Rev, Chil, Pediatr. 56(2) : 83-86, 1985

\title{
Preparación de Solución Hidratante Oral en el Hogar.
}

\author{
Dr. Francisco Olivari P.2; Dr. Gastón Dulfau T.1; \\ Q.F. Sra. Julia Hormazábal V.1
}

\section{Preparation of Oral Rehydration Solutions at Home}

In order to ascertain the convenience of setting up a program for oral rehydration with solutions prepared at home, 100 mothers attendíng an out patient clinic located in a low income urban area were randomly assigned to two groups: "Group I" received precise orai (spoken) instruction: "Group 2" received the same kind of instructions plus practical demostrations about the procedure to be employed. Both groups of mothers were requested to dilute $20 \mathrm{~g}$. of glucose contained in a small plastic sealed envelope into one liter of water in order to obtain a $11 \mathrm{l} \mathrm{mmol/1.}$ solution. In $95 \%$ of the cases, the expected concentration $\pm 20 \%$ was obtained, this range being considered safe. In the remaining $5 \%$ excesively diluted samples were found, representing an eventual decrease in effectivity but not a real risk for patient's health. The diftetences in glucose concentration were not related to maternal age, nor degree of literacy neither the number of children within the family. The importance of complementing oral information with practical demonstrations is stressed since the variance analysis showed a significantly lesser dispersion of glucose concentration values in the solution prepared by mothers of "Group 2 " ( $\mathrm{p}<0.05$ ), in spite of very simila mean glucose concentrations from both groups. A program of oral rehydration with home prepared solutions appears as a feasible alternative in the treatme;t of acute dianthoeal disease in primary health care under the conditions of this study.

(Key words: Home-made mixtures. Oral rehydration solutions. Parent's education).

La utilización de terapia hidratante oral, empleando la solución propuesta por la Organización Mundial de la Salud (OMS) ${ }^{1}$, ha demostrado sus beneficios en el síndrome de diarrea aguda del adulto, del niño y del recién nacido, inde: pendientemente de su etiología, en numerosos

1 Departamento de Pediatria, División Ciencias Médicas Norte. Facultad de Medicina, Universidad de Chile, Hospital Roberto del Río.

2 Departamento de Pediatria, División Ciencias Médicas Norte, Facultad de Medicina, Universidad de Chile, Consultorio La Pincoya. paises $1 \cdot 2 \cdot 3-4-5-6-7$.

En nuestro medio, la entrega de sales para diluir en el hogar, fue abandonada hace unos 20 años, porque entre los lactantes hospitalizados con deshidratación hipernatrémica, el antecedente de ingestión de suero era más común que en los normonatrémicos o hiponatrémicos ${ }^{8-9}$. Esto coincidía con la observación que las sales entregadas a las madres, solian ser disueltas en un volumen inferior al indicado.

En los últimos años, hemos estudiado la aplicación de terapia hidratante oral con una solución pre-disuelta, en lactantes hospitalizados 
con deshidratación y en tratamientos de mantención, buscando la composición más conveniente $\mathrm{e}^{5-6}$.

Recientemente investigamos Ja posibilidad de entregar una solución, en la forma de sales por diluir, para conocer el nivel actual de error en el procedimiento, sin mediar entrenamiento alguno y determinar los contenidos educativos capaces de minimizarlo ${ }^{10}$.

Ahora presentamos un intento para aplicar la información obtenida, detectar posibles nuevos factores de error y buscar la forma más adecuada de entregar los contenidos educativos, comparando la mera instrucción con la demostración práctica.

\section{MATERIAL Y METODO}

El estudio se efectuó en el consultorio La Pincoya, perteneciente al Servicio de Salud Metropolitano Norte, durante el mes de Enero de 1984.

Se tomaron dos grupos al azar ( 1 y 2 ), de 50 madres cada uno, cuyos hijos estaban en control en el consultorio y en ambos casos se les pidió ta preparación de una solución en el hogar, usando $20 \mathrm{~g}$. de glucosa envasada en sobres de polietileno, para disolver en I litro de agua, en otras palabras una concentración de $11 \mathrm{l} \mathrm{mmol} / \mathrm{l}$.

Uno de nosotros (F,O.), instruyó verbalmente $y$ en forma precisa, sobre la forma más adecuada de preparar la solución a las madres del Grupo 1. En el Grupo 2, se efectuó además una demostración, destacando aquello que pudiera evitar errores en la dilución.

En las indicaciones se puso énfasis en que se utilizaran sólo botellas comerciales de bebidas gaseosas de l litro, cuyo volumen se encuentra impreso y claramente visible en el vidrio; que la apertura del sobre fuera realizada con tijera y que se usara un embudo al vaciar el contenido, para evitar su derramamiento.

En todos los casos se tomó una muestra de la formulación obtenida, estabilizándola con ácido benzoico, para medjción ulterior de la concentraciốn de glucosa. Se emplearon frascos de cierre hermético en el transporte. El análisis se hizo en el Laboratorio de Investigación del Departamento de Pediatría del Hospital Roberto del Río, mediante el método de Somogy--Nelson.

En cada caso se obtuvo información sobre disponibilidad de medidas de un litro para realizar la dilución, forma en que se efectuó el procedimiento y problemas que se presentaron al hacerlo. También se consignó edad y escolaridad de la madre y nútnero de hijos, con el objeto de comparar los grupos de estos parámetros.

Se fijó en forma arbitrarja un margen de error aceptable de un $20 \%$, lo que significa concentraciones de glucosa entre 88,8 y $133,2 \mathrm{mmol} / \mathrm{l}$. Este margen es más exigente que el considerado adecuado por la OMS, para soluciones preparadas en el hogar ${ }^{1}$.

Los resultados numéricos fueron sometidos a pruebas estadisticas de significación $(\mathrm{t}$. de Student, prueba $Z$, análisis de varianza y $\left.\mathrm{X}^{2}\right)^{11}$.

\section{RESULTADOS}

Ambos grupos resultaron perfectamente comparables y con una estructura similar a la detectada de una experiencia anterior, efectuada en la misma población ${ }^{10}$ (Tabla 1 ).

Tabla 1.

Preparación de solución hidiratante oral en el hogar. Concentración de glucosal obtenida en mmolil.

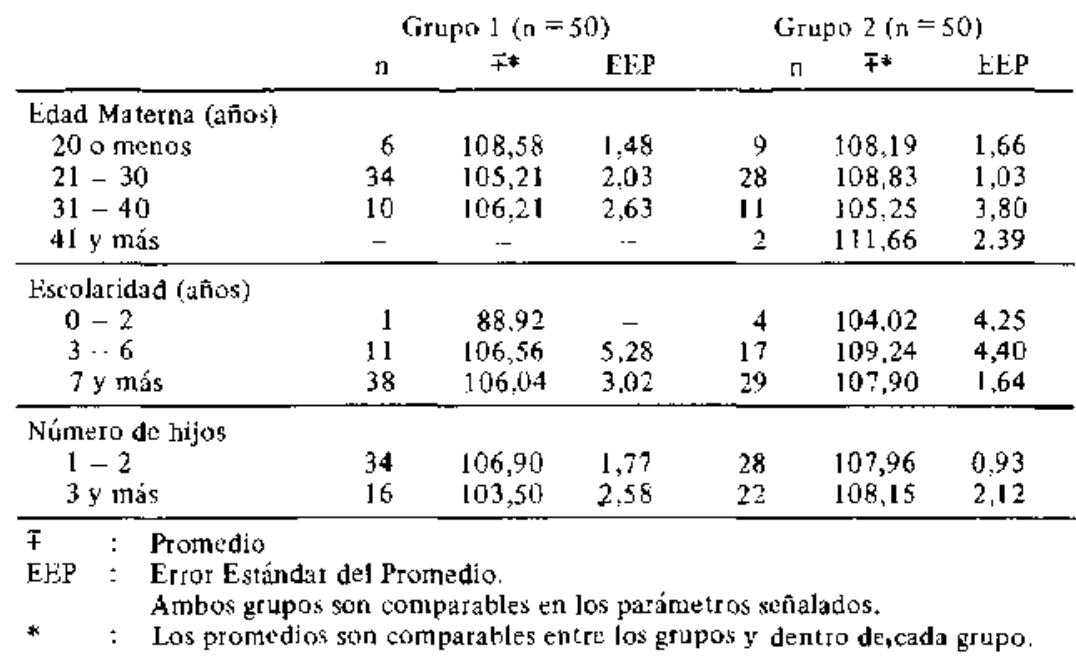


Las recomendaciones suministradas a las madres participantes de la investigación fueron, en general, seguidas cuidadosamente: Todas manifestaron haber empleado envases de un litro, porque disponian de él en casa, o no tuvieron dificultad para conseguirlo.

La apertura del sobre de polietileno no fue tan simple y la utilización de tijeras y embudo para evitar el derramamiento de soluto no fue constante.

El tiempo transcurrido entre la preparación y la estabilización de la muestra, fue de 1.85 horas, promedio.

La concentración de glucosa promedio en el total de las muestras, fue $106.9 \mathrm{mmol} / \mathrm{l}$, con un margen de 64.3 a 125.9 mmol/1. Esto significa que el error, considerando el ideal esperado de $111 \mathrm{mmol} / 1$, fue de $13,4 \%$ más hasta $42 \%$ menos.

La edad y escolaridad de la madre y el número de hijos, no mostraron influencias sobre los valores centrales de concentración de glucosa obtenidos (Tabla 1).

En $95 \%$ de los casos la concentración del preparado, estuvo dentro del margen definido como seguro. En el $5 \%$ restante se encontraron diluciones excesivas ( 4 casos en el Grupo 1 y 1 caso en el Grupo 2).

Al evaluar el efecto de la instrucción verbal a las madres, aislada y conjuntamente con una demostración sobre lo solicitado (Tabla 2), se puede señalar que los promedios de concentración de glucosa obtenidas, resultaron comparables, pero en el Grupo 2, se encontró una dispersión significativamente menor de las cifras.

Tabla 2.

Preparación de solución hidratante oral en el hogar. Concentración de Glucosa obtenida en mmol/l.

\begin{tabular}{lcccc}
\hline & $\mathrm{n}$ & $\mp$ & $\mathrm{EEP}$ & $\mathrm{V}$ \\
\hline Grupo 1* & 50 & $105,8 \#$ & $\mathbf{1}, 48$ & $110,2 / /$ \\
Grupo 2* $^{*}$ & 50 & $108,0 \#$ & 1,05 & $55,8 / 7$ \\
\hline Total & 100 & 106,9 & 0,91 & 83,3 \\
\hline
\end{tabular}

\begin{tabular}{lll}
\hline EEP & $:$ Error Esstándard del Promedio \\
$\mathrm{y}$ & $:$ Varianza \\
$*$ & $:$ Ei Grupo 1 recibió instrucción verbal y \\
& el Grupo 2, además, una demostración \\
$\# \quad: \quad \mathrm{p}>0,05$ \\
$\Rightarrow \quad: \quad \mathrm{p}<0,05$
\end{tabular}

\section{COMENTARIO}

En el empleo de una solución hidratante oral, en la forma de sales para disolver, en el manejo ambulatorio del sindrome de diarrea aguda, nos enfrentamos al prablema del error que se produce en el proceso de preparación en el hogar ${ }^{8-9}$ Este depende fundamentalmente de la medición del volumen necesario para diluir la cantidad ya medida de soluto y producir una solución sin riesgos, para ser usada básicamente en el tratamiento de mantención $\mathrm{y}$ en la prevención de deshidratación en la diarrea agudal.

Una buena manera de resolver el problema, es disponer de una medida unjversal del volumen del líquido que debe emplearse y dar a la madre instrucciones adecuadas para realizar la dilución.

Los resultados obtenidos en ambos grupos se consideran excelentes. La pequeña proporción de errores observados, consistió en la dilución excesiva de la preparación, lo que no sepresenta un riesgo mayor en la utilización de la solución. Sin embargo, parece necesario instruir mejor, para reducir el derramamiento de soluto.

Se corroboró lo observado anteriormente ${ }^{10}$ en que las concentraciones centrales obtenidas con el procedimiento, no se vieron afectadas por la edad y la escolaridad maternas, ni por el número de hijos.

Un hecho importante, es que todas las madres dispusieron y utilizaron envases comerciales de un litro, que se acercan bastante a constituir una medida relativamente universal en los hogares, que permite minimizar las probabilidades de error. A mayor abundamiento, en la actualidad, es posible encontrar en el comercio más de veinte tipos distintos de envases de un litro, que contienen productos de consumo habitual y traen impresa la capacidad.

Al analizar los resultados, según la forma en que se entregó la información a las madres, se aprecia la importancia de realizar demostraciones del procedimiento. Si bien, las cifras promedio de concentración fueron similares, la diferencia observada en la dispersion fue significativa, siendo ésta menor en el grupo que recibió las instrucciones con este método. Es interesante señalar que para un margen de error establecido arbitrariamente en $20 \%$, la proporción de casos que se salió de éste es comparable: $8 \%$ en el Grupo 1 y $2 \%$ en el Grupo 2 ( $p>0.05$ ); de modo que la decisión de efectuar o no demostraciones, estará condicionada por el grado de seguridad que se estime conveniente establecer.

En consideración a la pequeña magnitud del error y la tendencia hacia la preparación de soluciones más diluídas que las esperadas en la preparación de soluciones hidratantes orales en el hogar, si se entregan instrucciones adecuadamente, es razonable pensar que este elemento puede incorporarse al tratamiento ambulatorio del sindrome diarreico agudo en nuestro medio. Mencionaremos que, sin mediar programa alguno 
de entrega de solución glucoelectrolítica balanceada para prevención de la deshidratación o terapia de mantenimiento, la mortalidad por diarrea aguda en nuestro pajs, es rełativamente baja, si se compara con lo encostrado en muchos otros $^{12}$, lo que hace necesario realizar estudios como éste para asegurar que la eventual morbilidad derivada del mal empleo de las soluciones orales en el hogar, no exceda los posibles beneficios.

\section{RESUMEN}

Para determinar la conveniencia de entregar una solución hidratante oral - en la forma de solutos por diluir-. en el manejo del síndrome diarreico agudo en el hogar y establecer la forma más adecuada de entregar los contenidos educativos, se estudiaron dos grupos de 50 madres cada uno, det consultorio La Pincoya, comparando la mera instrucción (Grupo 1) con la demostración práctica (Grupo 2)

Se proporcionó a las madres, glucosa en sobres de polietileno conteniendo $20 \mathrm{~g}$, para ser disuelta en 1 litro de agua y generar una concentración de $111 \mathrm{mmol} / 1$.

El $95 \%$ de las muestras estuvo dentro del margen definido como de seguridad (cifra esperada $\pm 20 \%$ ). Las preparaciones erróneas, correspondieron exclusivamente a diluciones excesivas, lo que representaria una eventual disminución en rendimiento, pero no un riesgo mayor.

La composición de las soluciones obtenidas, no varió con la edad y escolaridad materna, ni con el número de hijos.

Se destaca la importancia de suministrar los contenidos educativos, recurriendo a la demostración, ya que si bien los grupos l y 2 no mostraron diferencias significativas desde el punto de vista de los promedios de concentración de glucosa logrados, las varianzas señalaron que el segundo tiene una dispersión de valores claramente menor $(p<0.05)$.

Se comenta la posibilidad de incorporar el uso de una solución hidratante oral, en forma de sales para disolver, en el manejo ambulatorio del sindrome diarreico agudo en nuestro medio.

\section{REFERENCIAS}

1 Wortd Health Organization. The matuagencit of diarrhea and use of oral rehydration therapy. A join WHO/UNICEF statement. Genova, 1983.

2 Dartow, D., Pratt, E., Fleit, J., Gamble, A., Wiese, H.: Disturbance of water and electrolytes in infants diarrhea. Pediatrics, 3; 129, 1949

${ }^{3}$ Hirschhorn, $N$.: The treatment of acute diartica in children: an historical and physiological perspective. Am. J. Clin. Nutr. 33:637, 1980.

4 Brow", X.H. Mclean, W.C.: Nutritional management of acute diarshea: An appraisal of the alternatives. Pediatrics 73: 119, 1984 .

5 Duffau, G. Emilfork, M., Colderón, A.: Evaluación de dos fórmulas para hidratación oral en el síndrome diarteico agudo con destuidratación en el lactante. Bol. Med. Hosp. Intant. Mex. 39, 11; 729, 1982.

${ }^{6}$ Emilfork, M., Duffau, G.: livaluación de una solución on la terapia de mantención del síndrome diarreico agudo sin deshidratación del lactante. Bol. Med. Hosp. Infant. Mex. 40, 3: 135, 1983.

7 Hirschhorn, N.: Westly, T.: Oral rehydration of chitdren with acute diarthea. Lancet 2: 494, 1972.

${ }^{8}$ Soriano, H., Agiilo, C.. Macaya, J., Duffau, G. Colderón, A.: Síndrome diarreico agudo con deshiđratación del lactante. Correlación clínico humoral. Pediatría, (Stgo.) 15:425, 1972

${ }^{9}$ Colle, E., Ayoub, E., Ralle, R.: Hypertonic dehydra. tion (hypernatremia). The role of feedings high in solutes. Pedaltrics 22: $5,1958$.

10 Molina, C., Duffai, G., Hormazábal, J.: Solución para hidaratación oral en sindrome diarseico agudo del niño. Ensayo de preparación es el hogar. Pedia. tria ( $\$$ tgo.) por publicarse.

11 La Morte, M. Fistadística biológica. Barcelona: liditorial Mason, 1965.

12 World Health Organization: Levels, trends, differeniials, and cauxes of infant and early childhoud mortality in Latin America. World Health Statistics Quarterly 32: 147, 1982. 